\title{
Smart EPU: SPA Getting Intelligent
}

Yuchen Deng ${ }^{1}$, Fanis Grollios ${ }^{2}$, Holger $\mathrm{Kohr}^{3}$, Bart van Knippenberg ${ }^{2}$, Michael Janus ${ }^{1}$ and Faruk Caglar ${ }^{3}$

${ }^{1}$ Thermo Fisher Scientific, United States, ${ }^{2}$ Thermo Fisher Scientific, Eindhoven, Netherlands, ${ }^{3}$ Thermo Fisher Scientific, Netherlands

Single Particle Analysis (SPA) is an increasingly popular technique among structural biologists for visualizing the structures of proteins and protein complexes [1,2]. Recent improvements in instrumentation and automation improve the efficiency with which high-quality data can be obtained, which will likely broaden the applicability [3] of this workflow to users that have limited microscopy expertise. However, acquiring high-quality SPA data still largely depends on the expertise of the microscope operator. This limits the speed of adoption as researchers must invest considerable time into understanding microscopy and the technicalities of the workflow. In this presentation, we introduce Smart EPU, a new technology on Thermo Scientific Cryo-TEM systems that allows for further software and ease of use automation. Smart EPU will free operators from tedious and repetitive tasks and open Cryo-EM to a wider audience by taking over tasks that are easy for experts but challenging novices.

Smart EPU [Figure 1] provides an open interface that enables smart algorithms to connect to an ongoing acquisition and optimize it for efficiency and quality by leveraging data and metadata produced by the microscope in real time. Our automation platform also ensures that decisions made by algorithms are applied effectively, without inter-algorithm conflict, and without compromising microscope stability. This platform integrates with our data management and processing infrastructure and besides build-in algorithms it will remain open for custom plug-ins build by community members.

We present our first set of algorithms that connect to smart EPU. They digest the results of processing routines such as motion correction, and CTF determination (integrated in Thermo Scientific EPU Quality Monitor [4]), to make on the fly smart decisions that optimize the session. These algorithms remove the necessity of the autofocus procedure, and automatically adjust stage waiting time based on the microscope condition. Additionally, by leveraging the power of AI, these algorithms encode the knowledge of microscopy experts and can automatically move on from data collection on sample areas of poor quality. This frees up scientists from having to predefine the perfect setup for quality data acquisition as the algorithms will refine their initial setup conditions on the fly, informed by the real time data coming off the microscope. 


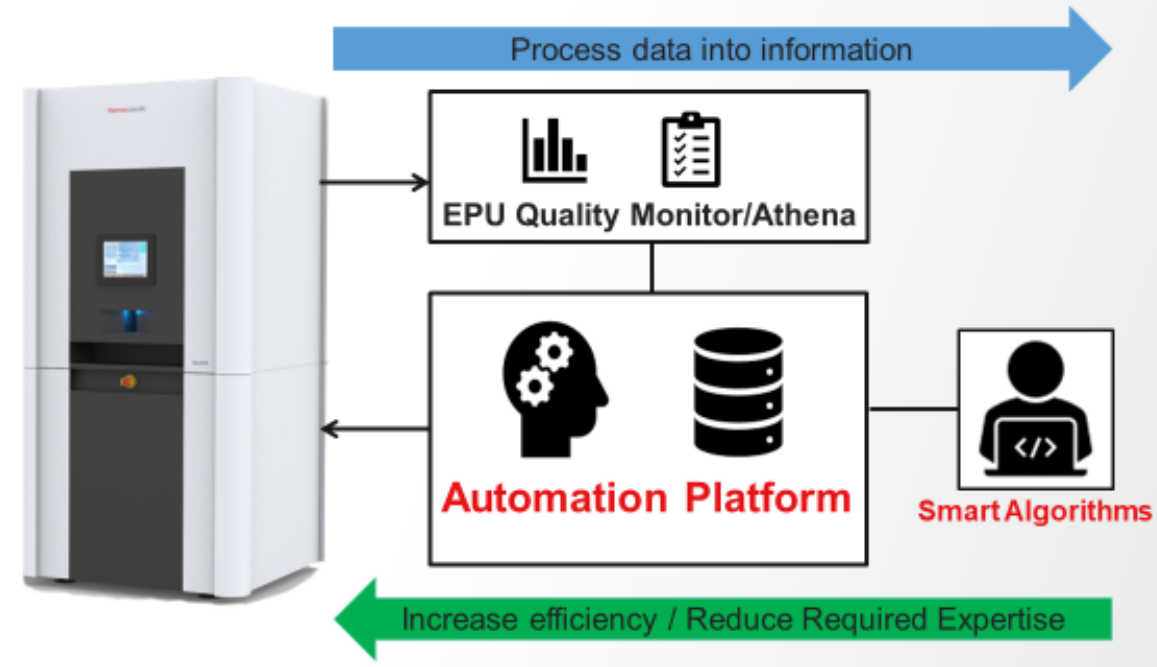

Figure 1. Overview of Smart EPU

\section{References}

[1] The Nobel Prize in Chemistry 2017, Jacques Dubochet, Joachim Frank, Richard Henderson

[2] Nature Methods, Special feature: Method of the Year 2015 (2015)

[3] Single-particle cryo-EM at atomic resolution, Nakane, T., Kotecha, A., Sente, A. et al. Nature 587, 152-156 (2020)

[4] Thermo Fisher Scientific, EPU 2 Software, last accessed February 2021

$<\quad$ https://www.thermofisher.com/nl/en/home/electron-microscopy/products/software-em-3d-vis/epusoftware.html > 